\title{
A Modified Genetic Algorithm for Optimal Allocation of Capacitor Banks in MV Distribution Networks
}

\author{
Antonino Augugliaro $^{1}$ - Luigi Dusonchet ${ }^{1}$ - Salvatore Favuzza ${ }^{1}$. \\ Mariano Giuseppe Ippolito $^{1}$ - Stefano Mangione ${ }^{1}$ - Eleonora Riva Sanseverino ${ }^{1}$
}

Received: 30 March 2015 / Revised: 10 July 2015 / Accepted: 14 July 2015 / Published online: 4 September 2015

(C) Springer Science+Business Media Singapore 2015

\begin{abstract}
In this paper, a genetic algorithm is developed in which each individual is represented not by a string but by a matrix: in this way the entire population is represented by a 3-D matrix. Such a representation is particularly useful for solving optimization problems with many discrete variables: such as, for example, the optimal allocation and sizing of distributed generation systems or the optimal compensation in a distribution system through the installation, in a prefixed number of nodes, of batteries of fixed or modulated capacitors, having different sizes. In these cases, the unknowns of the optimization process are the nodes where the apparatus can be installed and their rated sizes to be chosen in a discrete set of values. The adopted representation allows setting up a group of new operators whose application implies a strong increase of the search space size and consequently the number of algorithm parameters increases as well. The primary objective of this paper is that of evaluating the algorithm's performance and, with this aim, it has been applied to the problem of the compensation of electrical distribution networks.
\end{abstract}

Keywords Distribution network compensation - Capacitor siting and sizing · Genetic algorithm

\section{Introduction}

In the last years distribution systems have changed a great deal, for a number of reasons, among which are market liberalization; the increased use of automation techniques,

Salvatore Favuzza

salvatore.favuzza@unipa.it; favuzza@dieet.unipa.it

1 DEIM, Università di Palermo, Viale delle Scienze-Edificio 9, 90128 Palermo, Italy information technology and telecommunications; the introduction of small generation systems both traditional and renewable; increasing attention to the improvement of the quality of supply; etc.

The influence of these factors has produced the need for significant changes in the design and operation criteria for such systems. In particular, the installation of capacitor banks allows, by reducing the reactive flows in the lines, reducing the power losses in the system, improving the bus voltage profile, reducing the thermal load on the conductors, and influencing the voltage collapse risk. Since the network loads change over time, in order to attain the cited benefits, it is necessary that the compensation system be able to modulate its own reactive power production. Such a goal can be attained through fixed or modulated capacitor banks. In this latter case the modulation is generally carried out in steps. The optimal design of the compensation system, namely the identification of the nodes where reactive power banks can be installed as well as their rated power, is a complex combinatorial problem, with discrete and non-linear variables having the objective of minimizing the costs connected to the losses and the installation of capacitor banks. In the literature, the most used solution methodologies use metaheuristic algorithms (genetic algorithms, tabu search, simulated annealing, ant colonies, etc.) that allow finding suboptimal solutions in reasonable calculation times; the quality of these solutions strongly depends on the algorithm's parameters.

\section{State of the Art}

The problem of capacitor allocation for loss reduction in electric distribution systems has been extensively researched over the past several decades. Ng et al. [20] have described the evolution, from 1970 until 2000, of this research and provided 
an evaluation of the practicality and accuracy of the capacitor placement algorithms in the literature of the period. The paper also provides researchers and utility engineers an insight into the choices between the available capacitor allocation techniques and their respective merits and shortcomings.

The same authors [21] have presented an approach using approximate reasoning to determine suitable candidate nodes in a distribution system for capacitor placement. Voltage and power loss reduction indexes of distribution system nodes are modelled by fuzzy membership functions. A fuzzy expert system (FES) containing a set of heuristic rules is used to determine the capacitor placement suitability of each node in the distribution system. Capacitors are then placed on the nodes with the highest suitability.

A practical solution technique to the capacitor placement problem is developed by Carlisle et al. [4]. The proposed approach uses a graph search algorithm. The method can handle standard capacitor sizes and associated costs, and the resulting non-differentiable objective function. It can also determine the type of capacitors to be installed, and the switching times of the switched ones. The objective function takes into account the reduction in peak power losses, the reduction in energy losses, and the total cost of the capacitors. In the development of the objective function, some simplifying assumptions are made: capacitor placement affects only the flow of reactive power in the feeder, the real power flow remaining constant; rated voltages are assumed at every point on the feeder, and are not affected by the addition of capacitors; only balanced three-phase loads are considered.

A method employing the immune algorithm (IA) is proposed by Huang [14] to solve capacitor placement problems. The objectives of capacitor placement are to reduce the energy loss and to maintain voltage levels within the allowable limits while minimizing the total system cost. The objectives and constraints are firstly represented as antigen inputs. This is followed by antibody production on a feasible space through genetic operations. Through the IA computation, an antibody that most fits the antigen is considered as a solution to the problem. Because the energy loss is critical to the placement performance, the load variations are taken into consideration for a given period of time. It is assumed that the loads vary in a conforming way and their variations are approximated with discrete levels.

A formulation for the optimal capacitor allocation problem is presented by Hsiao and Chien [13]. This formulation considers four different objective functions related to cost investment, operating efficiency, system security and service quality, which represent a priority issue for the system planner. An interactive trade-off algorithm is developed, based on the E-constrained technique to obtain a compromised or most satisfactory non-inferior solution.

A hybrid method drawn upon the Tabu Search approach is proposed by Gallego et al. [9], extended with features taken from other combinatorial approaches such as genetic algorithms and simulated annealing, and from practical heuristic approaches. The optimal capacitor placement problem determines the capacitor types, sizes, and locations, as well as the control schemes in order to correct the power factor, reduce losses, improve the voltage profile, and, in a more limited way, increase the circuit capacity under different load curve conditions and satisfying equipment and operating constraints. The objective function is formulated as the cost of the losses and investments over a period of time. Two types of capacitors are considered: fixed capacitors and switched (variable) capacitors whose taps can be changed according to the load level.

Mekhamer et al. [18] aims to review and to implement previous methods on the use of capacitor banks for reactive power compensation and voltage profile improvement. Comparisons are made between them in order to select the best one. To show the closeness or remoteness from the exact solution after implementing these methods, a new algorithm (based on heuristic ideas and fuzzy techniques) to get this optimal capacitor allocation is presented.

Masoum et al. [17] presents a genetic algorithm based approach for the simultaneous power quality improvement and optimal placement and sizing of fixed capacitor banks in radial distribution networks in the presence of voltage and current harmonics. The objective function includes the cost of power losses, of energy losses and of the capacitor banks. Constraints include the voltage limits, the number/size and locations of the installed capacitors, and the power quality limits. Candidate buses for capacitor placement are selected based on an initial generation of chromosomes. Using a proposed fitness function, a suitable combination of objective and constraints is defined as a criterion to select, among the candidates, the most suitable buses for capacitor placement.

A new method, based on evolutionary algorithms, is proposed by Mendes et al. [19], capable of solving large network instances that appear in real-world settings. The objective function of the problem takes into account the annual gain due to energy loss reduction and the cost of the capacitor installation. An option is also introduced to limit the number of installed capacitors. This constraint comes from operational restrictions related to the maintenance team. The evolutionary approach makes use of a memetic algorithm that employs a hierarchical organisation of the population in overlapping clusters.

Venkatesh and Ranjan [23] proposes a single dynamic data structure for an evolutionary programming (EP) algorithm that handles the problems of siting and sizing of new shunt capacitors simultaneously while considering transformer taps, existing reactive power sources and reconfiguration options, accounting for different load levels and time durations. The overall problem has multiple objectives of minimisation of the total cost of new capacitors and the 
minimisation of power losses in the lines while obtaining a satisfactory voltage profile. To handle the multiple objectives and constraints, the problem is translated into a fuzzy formulation. A fuzzy model of the objective function is developed for optimisation in the EP framework.

A new formulation including the robustness of the solution of a constrained multiobjective design of reactive power compensation is presented by Augugliaro et al. [3]. The issue of robustness is included due to uncertainty and errors in loads estimation. The algorithm used for optimization is the Non dominated Sorting Genetic Algorithm II (NSGAII) with a special crowded comparison operator for handling constraints.

A method for capacitor placement based on a genetic algorithm (GA) is presented by Haghifam and Malik [12]. The optimal placement of fixed and switchable capacitors is obtained in one stage. The aims of the capacitor placement include the improvement of the bus voltages, the minimisation of energy losses and the reduction of demand in the feeder. The method is applied to distribution systems with varying loads. Uncertainty in the load allocation process or load forecasting is overcome by a fuzzy presentation of the loads in the problem formulation.

A genetic algorithm, in conjunction with fuzzy logic, is proposed by Ladjavardi and Masoum [16] for simultaneous improvement of power quality and optimal placement and sizing of fixed capacitor banks in distribution networks with non-linear loads imposing voltage and current harmonics. The economic cost is defined as the objective function and includes the cost of power losses, of energy losses, and of capacitor banks while the voltage limits, number/size of installed capacitors at each bus, and the power quality limits of harmonic are considered as constraints. Fuzzy approximate reasoning is used to calculate the fitness function in order to consider the uncertainty of decision making based on the suitability of constraints and the objective function (cost index) for each chromosome. Based on the proposed coding of each individual solution, three genetic operators were set up: simple mutation and two types of crossover.

Khodr et al. [15] present the problem as the maximization of the savings produced by the reduction in energy losses and the avoided costs due to deferral of any investment in expanding the network. The proposed method selects the nodes to be compensated, as well as the optimal capacitor ratings and their operational characteristics, i.e. fixed or switched. After an appropriate linearization, the optimization problem is formulated as a mixed-integer linear problem.

A heuristic constructive algorithm for optimal capacitor placement in distribution systems is presented by Chaves da Silva et al. [5]. In the proposed approach, a sigmoid function is used to approximate the discrete nature of the capacitor placement decision. Then the problem is formulated using the primal-dual interior point method. Two approaches to deal with sensitivities are proposed: (1) the number of candidate busbars is reduced based on a sensitivity index that is a multivariable function of the Lagrange multipliers, of the reactive power and of the voltage level; (2) a new heuristic technique based on the sigmoid function sensitivities and the reactive power is applied to the allocation decision. The objective function takes into account the cost of real power losses and the cost of capacitor banks.

Chang [6] proposes a method employing the ant colony search algorithm (ACSA) to solve the feeder reconfiguration and capacitor placement problems. The merits of the ACSA are its parallel search and optimization capabilities. This method is inspired by the observation of the behaviour of ant colonies. The ACSA used in the paper uses artificial ants, which have a memory to some extent and are not completely blind, and thus can be applied to the feeder reconfiguration and capacitor placement problems in which the switches are discrete. The state transition rule, and the global and local updating rules are introduced to ensure the optimal solution. The objective is to minimize the system power losses, subject to operating constraints under a certain load pattern.

The use of particle swarm optimization (PSO) is proposed by Eajal and El-Hawary [8] to solve the capacitor placement and sizing problem, which is formulated as a constrained nonlinear integer optimization problem with both the locations and the sizes of the shunt capacitors being discrete. The goal is to minimize the overall cost of the total real power losses and that of shunt capacitors while satisfying operating and power quality constraints (as the addition of shunt capacitors may lead to high distortion levels). A discrete version of PSO is combined with a radial distribution power flow algorithm (RDPF) to form a hybrid PSO algorithm (HPSO). The former is employed as a global optimizer to find the global optimal solution, while the latter is used to calculate the objective function and to verify the bus voltage limits. To include the presence of harmonics, the developed HPSO is integrated with a harmonic power flow algorithm (HPF).

An algorithm for the optimisation of radial distribution systems by a network reconfiguration and capacitor allocation is presented by Guimaraes et al. [10]. A modified, dedicated genetic algorithm based approach was developed and implemented. The objective function is formulated as the cost of the energy losses and the investments required for capacitor allocation over a period of time. The statuses of the sectionalising switches, and automatic and fixed capacitor information are stored in a single chromosome.

The optimal locations and sizes of capacitors with the objective of reduction of power loss and improving the voltage profile is developed by Abul'Wafa [1]. A loss sensitivity technique is used to select the candidate locations for capacitor placement. The size of the optimal capacitor at the compensated nodes is determined simultaneously by opti- 
mizing the loss saving equation with respect to the capacitor currents.

Attia et al. [2], used Artificial Bee Colony (ABC) for optimal capacitor placement problem with an objective to maximize the net savings per year and to improve the voltage profile. A teaching learning based optimization (TLBO) approach has been used by Sultana and Roy [22] to minimize power losses and energy cost by means an optimal placement of capacitors in radial distribution systems. Two bio-inspired algorithms have been implemented in [11] to solve optimal placement of capacitors in radial distribution systems in two ways: optimal placement of fixed size of capacitor banks and optimal sizing and placement of capacitors for real power losses minimization and network savings maximization.

In this paper a new genetic algorithm, in which the entire population is represented by means a 3-D matrix, is proposed and applied to solve the problem of the optimal allocation of capacitor banks, aiming at minimizing the costs of investments and power losses.

\section{Solution Strategy for the Problem of the Optimal Design of the Compensation System}

The issue of the optimal design of the compensation system, as discussed in this paper, is to identify, for a network with time-varying loads, the nodes where capacitor banks can be installed, their type (whether fixed or modulated in steps), and their rated power.

The objective is to minimize the total cost due to energy losses and the annualized installation cost of the capacitor banks. For the loads, modelled as constant power, the simplifying assumption that they vary conformally has been made, and therefore their course can be described by the duration curve.

Since either fixed or switchable capacitor banks can be installed, the solution strategy has been divided into two phases. The first (Sub-problem F) identifies:

- the nodes where capacitor banks are to be installed, and

- for each of the compensated nodes, an initial value of power of capacitor bank that over the whole period minimizes the Objective Function (OF).

In the second phase (Sub-problem S) there is identified, taking into account capacitive power just calculated for the compensated nodes, for such nodes and for each of the time periods in which the year is divided,

- the power of the switchable banks that minimize the OF showing a similar expression of the one minimized in the first phase.
In particular, the duration curve is divided into a finite number $m$ of time intervals and the shape of the curve is approximated, in each interval, by means of its average value; so, one obtains, for each compensated node, $m$ values of reactive power; the maximum capacitive value is added to capacitive power determined in the first phase, while the maximum inductive is deduced from capacitive power determined in the first phase.

For sub-problem $\mathrm{F}$ the reactive (capacitive) powers, $Q_{c, i}$, determined considering all the time intervals, both those with low loading and those with heavy loading, represent:

- for the highest loading condition, the minimum value ${ }^{1}$ of the power that can be installed at the nodes, to which the reactive power generated by the modulated banks can be added;

- for a loading condition below the maximum, such values can either be increased or decreased.

A reduction can be needed in those time intervals in which the loading is very low compared to the maximum and thus there exists the possibility that, as an effect of the values of the powers of the banks, the reactive power changes its nature from inductive to capacitive. In the same way, an increase can be required in those time intervals in which the loading is close to the maximum loading. In all cases, the optimization of sub-problem $\mathrm{S}$ is carried out taking into account in the compensated nodes the reactive power, $Q_{c, i}$, evaluated for the entire year (results of sub-problem F); the reactive power values calculated in each single time interval show thus differential values. As a result, for the $i$ th compensated node, $1+m$ values of reactive power are evaluated: the one relevant to the optimization over the whole year, $Q_{c, i}$, and those related to the load level in each of the time intervals $\tau=1,2, \ldots, m, Q_{c, i ; \tau}$ ( $\tau=1$ relates to the maximum loading and $\tau=m$ to the minimum loading). The rated power of the fixed battery is given by $Q_{c, i-f i x}=Q_{c, i}-$ $Q_{c, i m}$, while the power of the modulated part is given by $Q_{c, i-v a r}=Q_{c, i m}+Q_{c, i-1}$.

The partition of the switchable part in steps is carried out based on the remaining periods of time, in which the loading gets intermediate values; during the whole optimization

\footnotetext{
1 The optimization made in the first step determines, for each node, a value of the capacitive reactive power that is the same for all load levels; this value is therefore influenced by the values assumed by the load in all the periods and the length of these periods. The optimization made only for the period of maximum load, not considering the influence of the periods with lower load, gives a value of capacitive power that is surely higher. Similarly, for the period of minimum load, the capacitive power determined on the basis of the levels at higher load, is higher than the optimum and it is therefore necessary to reduce it. Obviously, the final decision to increase (for high loads) or reduce (for low loads) the capacitive power to be installed also depends on the sizes of the existing banks and the cost parameters.
} 
process, for capacitor banks the existing commercial sizes are considered.

In conclusion, the problem solution is reached by dividing it into two sub-problems to be solved one after the other:

Sub-problem F: Identify the nodes and the capacitive powers of the banks for the whole year based on the set of discrete values that approximate the duration curve of each load;

Sub-problem S: For all levels of power of the duration curve, taking into account both the compensated nodes and the relevant capacitive powers (attained through the solution of sub-problem F), identify the powers to be added or subtracted, by means of switchable capacitor banks. In this case, the yearly cost to assign to the additional banks is that of the switchable banks, in the case of addition of power, but it is the difference between the costs of the switchable and of the fixed banks, in the case of reduction. So, for each compensated node, the capacitive power totally installed, partitioned between the fixed and the switchable banks, and the partition in different steps of the switchable part are determined.

\section{Objective Function and Constraints}

The aim of the optimization process is to minimize the total yearly cost of the energy losses and of the cost of the capacitor banks. The loads vary conformally and the duration curve has been approximated with $m$ discrete values of the loading respectively lasting $T_{1}, T_{2}, \ldots, T_{m}$.

For the sub-problem $\mathrm{F}$ the objective function is expressed by:

$O F=k_{E}\left[\sum_{m} T_{j}\left\{\sum_{B} R_{i} I_{i, j}^{2}\right\}\right]+\sum k_{c f, k} Q_{c f, k}+\sum k_{F}$

where $k_{E}$ is the cost of energy, $\$ / \mathrm{kWh}, k_{c f, k}$ is the yearly cost of the fixed battery of capacitor banks with rated power $Q_{c f, k}$, $\$ / \mathrm{kVAR}, k_{F}$ is a fixed cost associated to each compensated node that does not depend on the power or the type of bank, $B$ is the set of branches of the network, $R_{i}$ is the resistance of branch $i, I_{i, j}$ is the current flowing in the $i$ th branch as the result of: (i) the network loads in the time interval $j$ lasting $T_{j}$; (ii) capacitor banks at network buses. Network is solved through Newton-Raphson method taking into account, for each node, real and reactive load, and capacitive power of the bank (if there is). In this way values of bus voltages and branch currents are available for testing constraints and $Q_{c f, k}$ is the capacitive power of bank at node $\mathrm{k}$.

The constraints here considered govern:

- the maximum current that can flow in the branches;

- the maximum and minimum voltages at the nodes;

- the uniform loading of the HV/MV transformers supplying the system;

- the maximum number of compensated nodes.

For sub-problem $\mathrm{S}$ the objective function in the generic time interval $T_{j}$ in which the year is divided, is

$$
O F_{j}=k_{E} T_{j}\left[\sum_{B} R_{i} I_{i, j}^{2}\right]+\sum k_{c s, k} Q_{c s, k}+\sum k_{F}
$$

where the symbols different from those appearing in (1) have the following meaning:

- $k_{c s, k}$ is the cost, related to the time $T_{j}$, to be given to the switchable bank at node k with rated power $Q_{c s, k, j}$, \$/kVAR; such cost equals the cost of the battery when the reactive power $Q_{c s, k, j}$ is capacitive (switchable bank to be added to fixed bank), but equals the difference between the cost of the switchable bank and that of the fixed bank when the reactive power $Q_{c s, k, j}$ is inductive; in this case, this is equivalent to the fact of correcting the value of power of the fixed bank calculated in sub-problem $F$. The total capacitive power to be installed at the node does not change, although the rated power of the switchable and fixed bank parts changes; the power $Q_{c s, k, j}$ thus represents the size of the switchable bank;

- $I_{i, j}$ is the current flowing in the $i$ th branch as the result of: (i) the network loads in the time interval $j$ lasting $T_{j}$; (ii) fixed capacitor banks at network buses; (iii) switchable capacitor banks at compensated buses. Bus voltages and branch currents are obtained as for sub-problem F.

The constraints considered are the first three of sub-problem $\mathrm{F}$, since the number of compensated nodes is fixed as a result of the solution of sub-problem $\mathrm{F}$, and a specific one referring to reactive power of compensated nodes that must be capacitive.

\section{Solution Methodology}

The considered optimization problem is NP complete with discrete variables and has a non-linear objective function and inequality constraints. For its solution, as suggested by most of the literature on the subject, a genetic algorithm suitably adapted to the problem at hand should be developed. Genetic algorithms (GA) are iterative methods simulating the evolution processes that take place in Nature, where a population of individuals (set of solutions) go through a set of genetic 


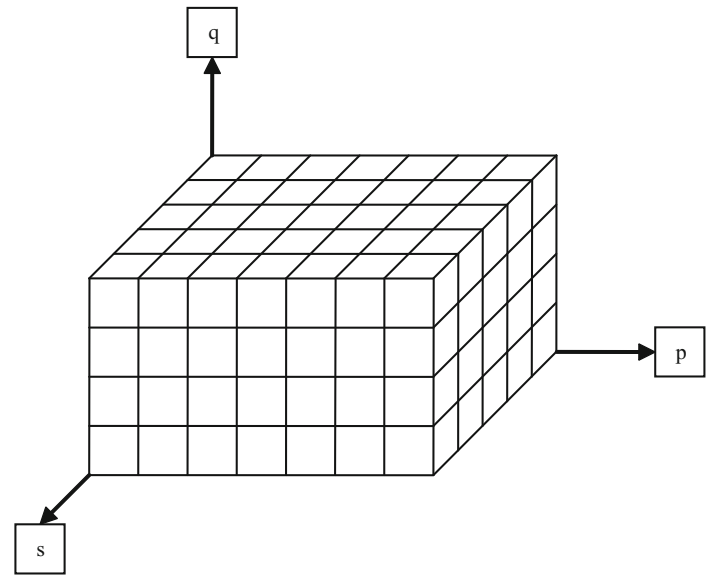

Fig. 1 Representation of population through a three-dimension matrix $|A| ; s=1,2, \ldots, N_{\text {cand }} ; p=1,2, \ldots, N_{\text {pop }} ; q=1,2, \ldots, N_{\text {mod }}$

recombinations. Each individual is evaluated based on a fitness function at each generation on the basis of the objective function and the constraints.

The possibility for an individual to survive is connected probabilistically to its quality.

To widen the search potential, operators that randomly modify randomly chosen individuals at each generation are introduced. The algorithm stops when a given convergence criterion is satisfied. GAs, just as other heuristics, are particularly efficient when the optimization problem has nondifferentiable objective functions and constraints.

Each solution is coded by a binary string. In this paper, the generic solution is represented by a matrix, $|\mathbf{I}|$, whose entry $I_{s, q}$ is 1 or 0 based on the presence or absence of a compensation module; the subscript $s$ indicates the node where a bank may be installed $\left(s=1, \ldots, N_{\text {cand }}\right.$ where $N_{\text {cand }}$ is the number of candidates nodes available for the installation of capacitor banks), and the subscript $q$ represents one of the possible sizes of the batteries $\left(q=1, \ldots, N_{\text {mod }}\right.$, where $N_{\text {mod }}$ is the number of modulation steps fixed for the banks).

Each generation is thus represented by a 3-D matrix, $|\mathbf{A}|$, with dimensions $N_{\text {pop }} \times N_{\text {cand }} \times N_{\text {mod }}$ (Fig. 1), whose entries $A_{p, s, q}$ are 0 or 1 and where the subscript $p$ is an individual of the population ( $p=1, \ldots, N_{\text {pop }}$, where $N_{\text {pop }}$ is the prefixed number of individuals making the starting population and the subsequent generations).

This representation has the benefit of unifying both locating and sizing problems as well as setting up a number of new perturbation operators, whose actions aim at improving the search potential of the algorithm, avoiding local minima/maxima.

For the specific problem of the compensation of a distribution system, the starting data are

- the electrical and topological features of the system (number of nodes, number of branches, number of HV/MV transformers, resistances and reactances of the branches, real and reactive powers of the loads);

- the candidate nodes for the installation of compensation banks;

- the maximum number of compensated nodes;

- the maximum number of modules of the banks and the reactive power of each module both for the fixed and for the switchable parts;

- the technical constraints on the bus voltages, branch currents, and power in the HV/MV transformers;

- the unit cost of energy and the yearly cost of the capacitor banks, both fixed and switchable;

- the diagram of the durations of the supplied loads represented through a histogram.

The main steps of the solution procedure are

\section{Sub-problem F}

(1) initial population generation;

(2) evaluation of the objective function and constraints for each solution;

(3) identification of the best individual;

(4) convergence criteria evaluation; in case of positive evaluation go to step 8

(5) production of the mating pool for the new generation creation through wheel roulette selection;

(6) random perturbation of some individuals through new operators 1-12 (see paragraph Genetic Operators);

(7) go to step 2.

\section{Sub-problem $S$}

(8) $\tau=1$;

(9) for the time interval $T_{\tau}$ into which the duration curve has been divided $(\tau=1,2, \ldots, \mathrm{m})$, evaluation of the reactive and real loads ( $\tau=1$ is for maximum loading and $\tau=\mathrm{m}$ the minimum loading);

(10) reactive power totally required at every compensated bus (difference between the reactive power required by the load and that of the fixed battery determined through the solution of sub-problem F);

(11) creation of the starting population: for all the individuals the compensated nodes are the same; the reactive power of the switchable banks are randomly chosen; their nature, capacitive or inductive, is determined based on a heuristic criterion. It is evaluated whether, considering the loads and the fixed banks reactive power, there is a reduction of energy losses upon increasing, or reducing, by one unit the reactive power of the existing banks ${ }^{2}$; one of the individuals keeps the features of the best solution found in sub-problem F;

$\overline{2}$ The economic convenience of switchable bank depends on the possibility to limit the energy losses; it is thus fundamental to verify whether 
(12) evaluation of the quality of each individual based on the objective function and constraints;

(13) identification of the best solution;

(14) evaluation of convergence criterion; if positive go to step 17 ;

(15) production of the mating pool through wheel roulette selection;

(16) random perturbation of some randomly chosen individuals through genetic operators $1-5,8,9$ (see paragraph Genetic Operators);

(17) $\tau=\tau+1$; if $\tau \leq$ m go to step 9;

(18) for each compensated node the reactive power of the fixed part is evaluated (difference between the value found at the end of sub-problem $F$ and that evaluated for $\tau=\mathrm{m})$ as well as the reactive power of the switchable part (summation of the powers evaluated for $\tau=1$ and $\tau=\mathrm{m})$

(19) stop.

\section{Starting Population}

In sub-problem $\mathrm{F}$, for each individual, the buses where the banks are to be installed are randomly chosen among the candidate nodes up to the maximum allowable number. For such nodes, for the two sub-problems $\mathrm{F}$ and $\mathrm{S}$ (in the latter the compensated nodes are known since they are identified by sub-problem F), the power of the modules in the compensated bus is chosen randomly through the attribution of a value 0 or 1 to the coefficient $a_{p, s, q}$ where $q$ varies between 1 up to the maximum number of modules considered for the capacitor banks ( $p$ is the index which is related to the individual, $s$ is the id of the compensated node).

\section{Genetic Operators}

The genetic perturbation operators set up and proposed in this paper can be considered as variants of the classical mutation and crossover, acting on one or more individuals, and adding new genetic material or combining existing genetic material. All the operators have been developed considering the constraint on the maximum number of compensated nodes; therefore the perturbations always give rise to feasible solutions. To improve the understanding of the way in which the different operators act, a set of figures are attached.

The mutation-like operators are the following:

(1) Simple mutation (Fig. 2) Based on a given probability, for the chosen individual one of the bits relevant to one

Footnote 2 continued

such reduction is connected to an increase or to a decrease of the power of the banks installed; of course the final choice depends on the cost parameters.

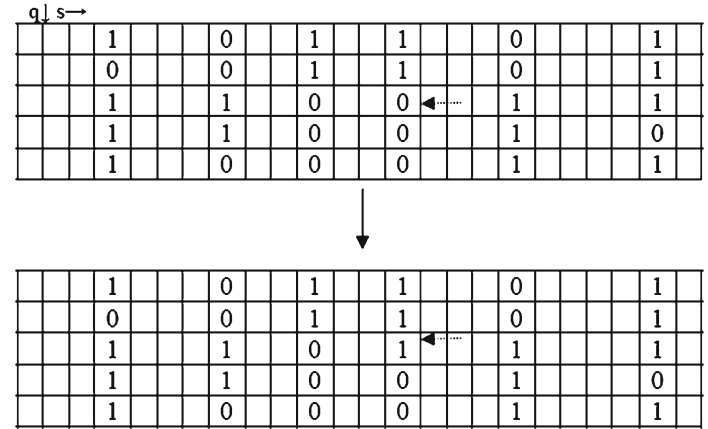

Fig. 2 Simple mutation

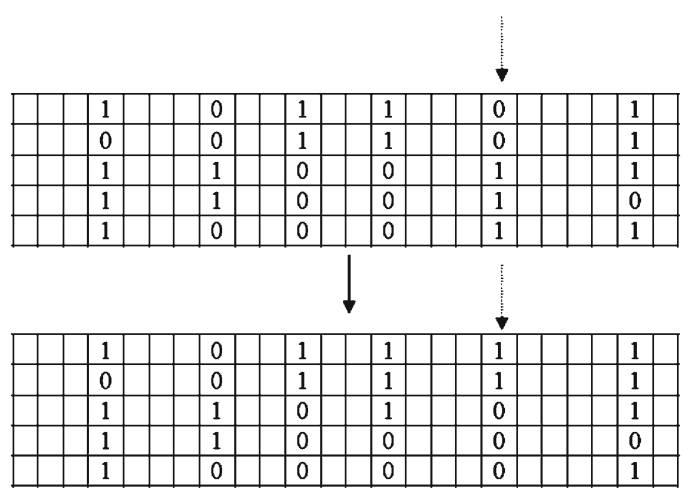

Fig. 3 Complete mutation

of the modules at the compensated node is modified; the effect is to modify the reactive power of the battery at the node. It may also happen that the reactive power of the battery is set to zero; this reduces the number of nodes that are compensated but satisfies the constraint on the maximum number of such nodes.

(2) Complete mutation (Fig. 3) This is applied to a compensated node of a randomly chosen individual and implies that, based on a given probability, a 1 is turned into 0 and vice versa of all the bits of the modules of the bank installed at the node; the reactive power at the node is thus strongly changed and, in some cases, it can be set to zero, hence reducing the number of compensated nodes.

(3) Simple mutation of a module (Fig. 4) Unlike the two preceding operators concerning one compensated node, this operator acts on all the compensated nodes of an individual; once one of the modules is chosen randomly, all the nodes have 0 turned to 1 and vice versa, based on a given probability. The effect sought is to significantly modify the distribution of the reactive powers of the batteries installed at the nodes; it may happen that the number of compensated nodes gets reduced.

(4) Complete mutation of the modules (Fig. 5) This is analogous to the preceding with the difference that, for the 


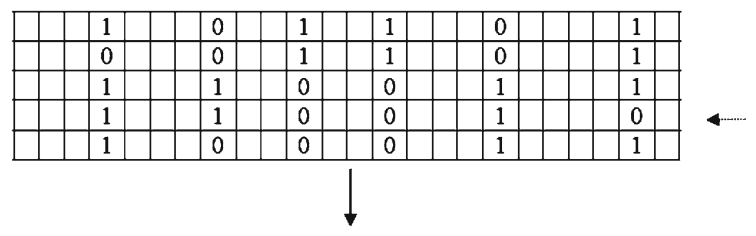

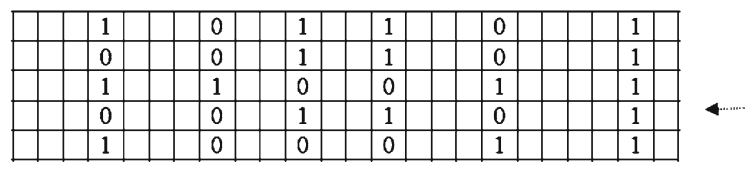

Fig. 4 Simple mutation of a module

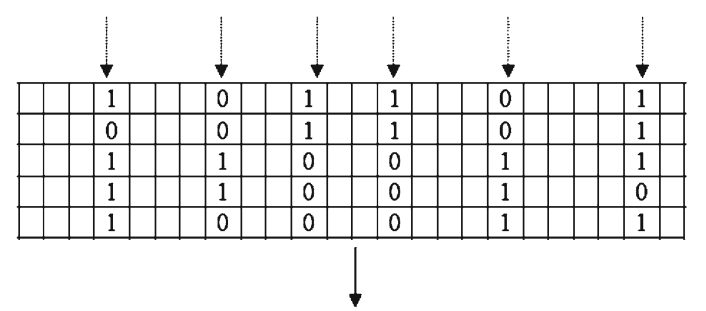

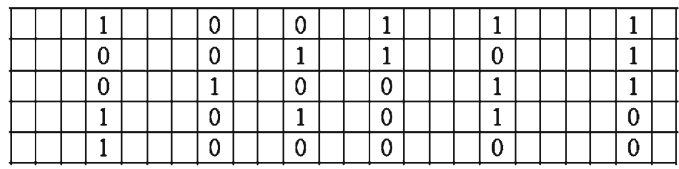

Fig. 5 Complete mutation of the modules

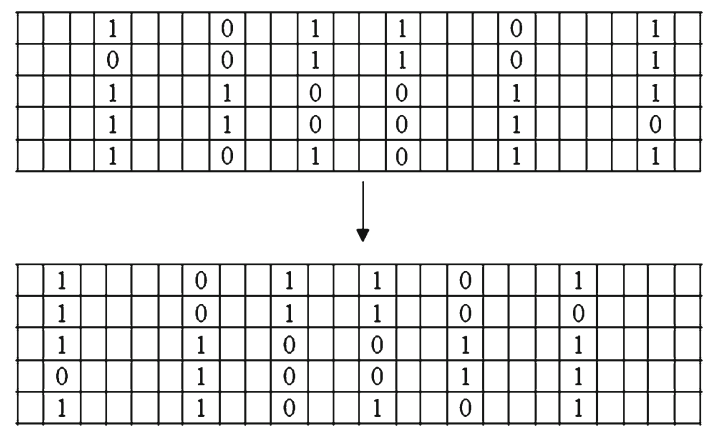

Fig. 6 Module inversion

considered individual, all the compensated nodes are subject to modification: for each compensated node and for each module it is decided, based on a given probability, whether the modification is executed or not. If yes, 1 is turned to 0 and vice versa. In this way the distribution of the reactive power of the capacitors at the nodes is significantly modified, with even a possible reduction of the compensated nodes.

(5) Module inversion (Fig. 6) For the chosen individual and for each of its compensated nodes, there is a given configuration of the power of the modules; the relevant binary string, which has as reference the first module, for each compensated node, is rearranged starting from the last module; in this way the total power installed in

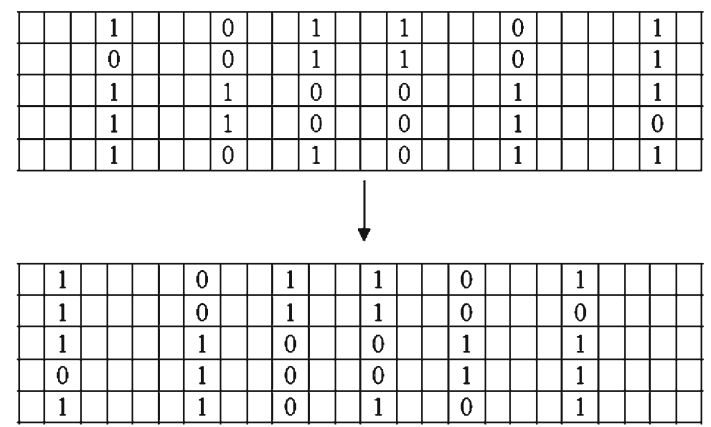

Fig. 7 Position inversion

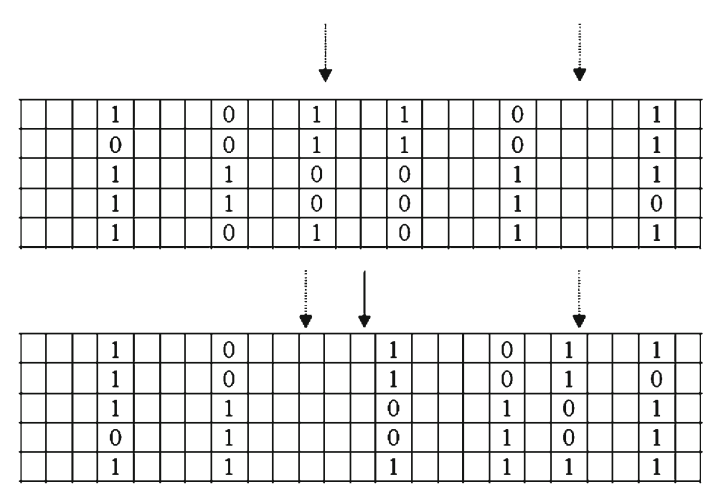

Fig. 8 Node exchange

the network is changed keeping the same positions and number of the compensated nodes.

(6) Position inversion (Fig. 7) The operators just described modify the power of the banks at the nodes keeping the same position; this operator changes the positions of the compensated nodes keeping the same power. In the individual chosen, the compensated nodes (as all the others) have an id and the source node has the lowest id; they are repositioned in the network taking as reference the highest id node; i.e., if the network has 80 nodes and in the starting configuration there is a bank at node 10 , after the mutation the same bank will be repositioned at node 70 .

(7) Node exchange (Fig. 8) With this operator the spatial distribution of the compensated nodes is modified; for each individual, based on a prefixed probability, two candidate nodes are randomly selected and the relevant reactive power features are exchanged.

(8) Module exchange (Fig. 9) This operator influences the total reactive power installed in a network modifying the power of the compensated nodes; once two modules are randomly chosen, for the chosen individual the binary strings of the two modules are exchanged. The crossover-like operators are the following:

(9) Module crossover (Fig. 10) Two individuals and a set of modules are randomly chosen, between the two 


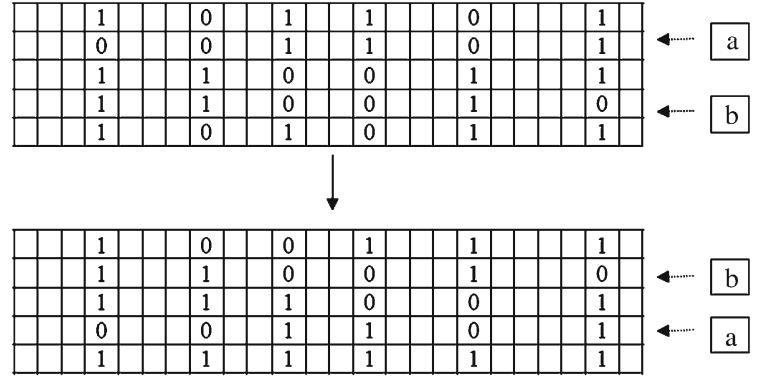

Fig. 9 Module exchange

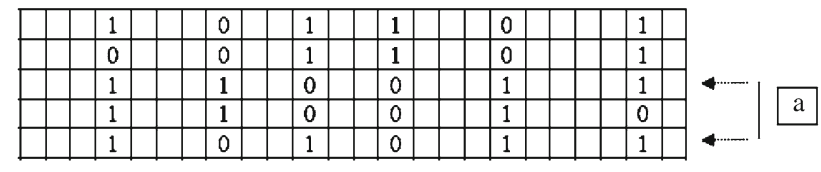

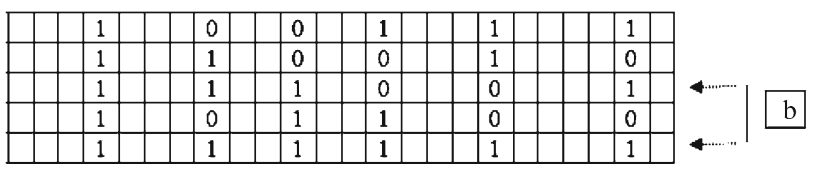

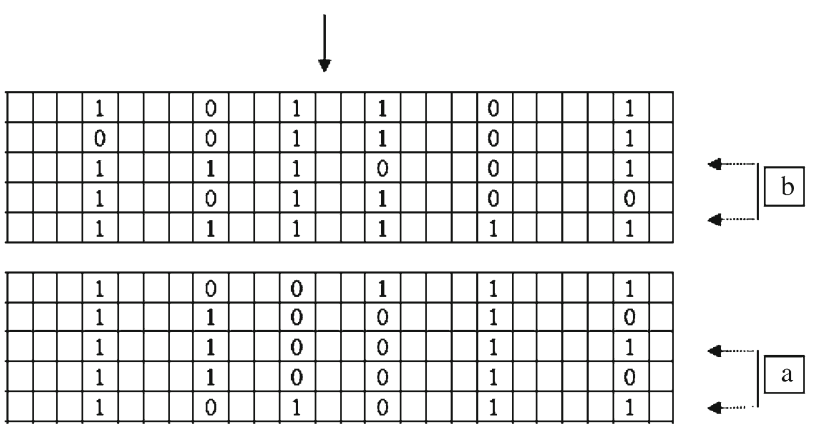

Fig. 10 Module crossover

individuals the modules of the set are exchanged; in this way, the two new individuals have the same spatial distribution of compensated nodes (with a possible increase or reduction of the compensated nodes, but satisfying the constraint on the maximum number of compensated nodes) $)^{3}$; the total reactive power of the two offspring solution changes, compared to the parents.

(10) Position crossover (Fig. 11) Two individuals are randomly chosen as well as an id between the possible

\footnotetext{
3 The operation executed on the matrices of the parents consists in the identification of a horizontal row relevant to a given number of modules. Each of the two offspring is attained building a matrix starting from one of the parents and putting in a sequence, in the missing parts of the columns, the modules that are in the other row; if the two parents show a number of compensated nodes equal to the maximum number, the two offsprings will therefore have a number of nodes that is equal or smaller; if one or both parents have a number of compensated nodes that is lower than the maximum, the two offsprings have a number of nodes that is smaller or equal to the maximum.
}

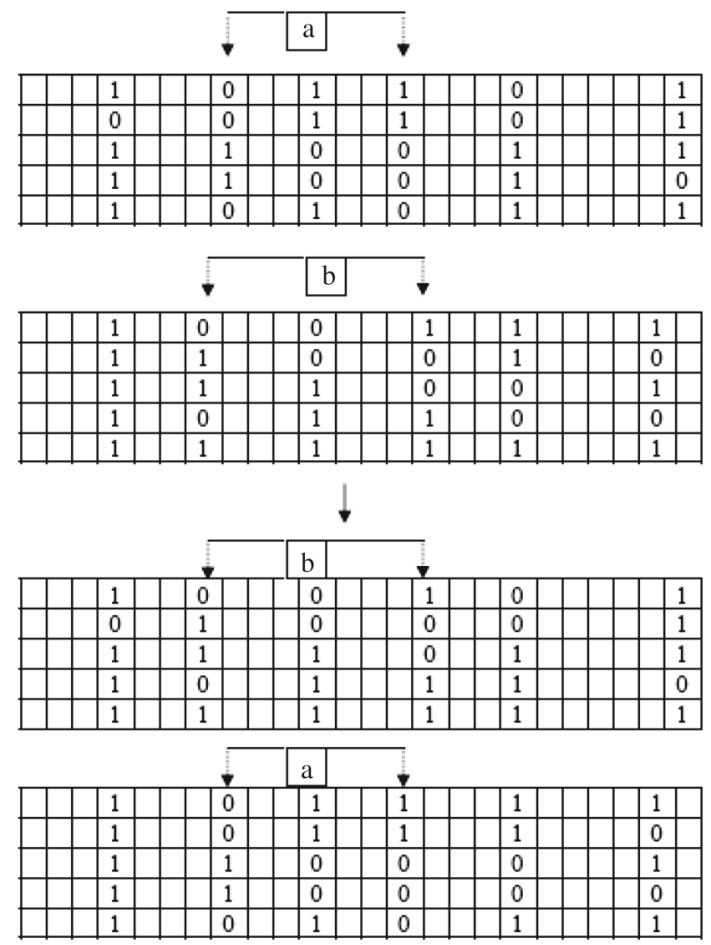

Fig. 11 Position crossover

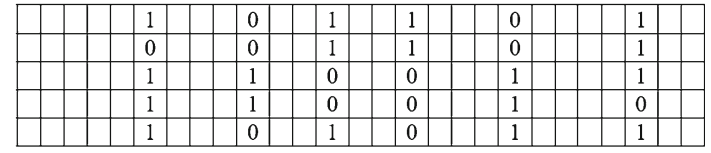

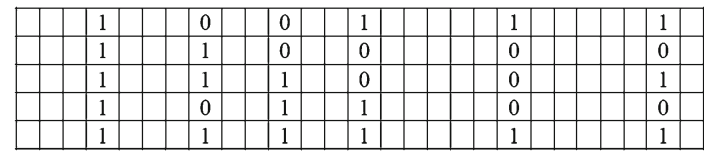

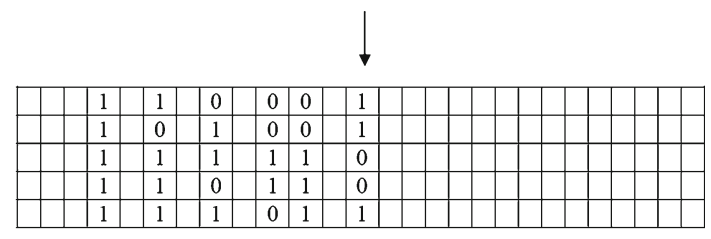

Fig. 12 Superposition from the initial bus

id of compensated nodes; the matrix representations of the two individuals are divided into two parts; the two new individuals are attained putting aside two different parts of the matrices corresponding to the two individuals. In the new solutions the maximum number of compensated nodes remains the same as before, but both the spatial distribution and the total reactive power of the banks change.

(11) Superposition from the initial bus (Fig. 12) Similarly to what was done before, this operator modifies the positions and the reactive power of the compensated nodes in the solutions it produces; unlike the above 


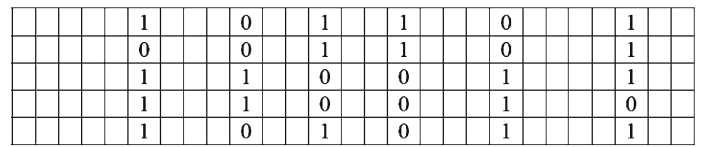

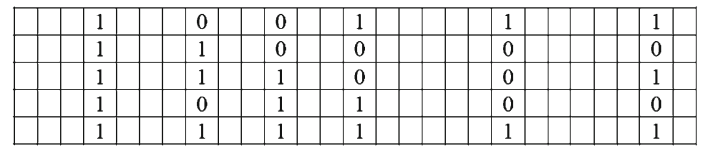

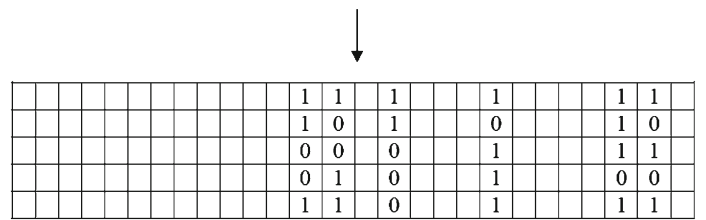

Fig. 13 Superposition from the ending node

operator, only one new solution is obtained. For each individual, another individual is randomly chosen and a new solution is generated, superposing the matrices of the two individuals starting from the node with the lowest id; in this way the compensated nodes of the two individuals are added to the new individual until the maximum number of compensated nodes is reached.

(12) Superposition from the ending node (Fig. 13) This operator acts in the same way as the preceding one with the difference that the superposition starts from the highest id node.

\section{Applications}

The procedure was implemented in a program, written in Fortran 90. The applications were carried out on a test system widely used in the literature; it is a $11-\mathrm{kV}$ radial distribution system having two substations, four feeders, 70 nodes, and 78 branches (including tie branches) as shown in Fig. 14. The tie switches of the system are open in normal conditions. The current carrying capacity of all tie branches are 234.0 A. Other topological data can be found in [7].

The loads vary conformally and their duration curve was approximated with three levels: for the low level the loading factor is equal to 0.7 with a duration of $2000 \mathrm{~h}$, for average loading it is equal to 1 with a duration of $3760 \mathrm{~h}$, and, for high loading, it is equal to 1.4 with a duration of $3000 \mathrm{~h}$. The cost of energy was set to $\$ 0.06 / \mathrm{kWh}$, while that of the capacitor banks was set to $\$ 5 / \mathrm{kVAR}$ and $\$ 6 / \mathrm{kVAR}$, respectively, for fixed and switchable capacitor banks, and independently of their size. For each compensated node a fixed cost of $\$ 1000$ was assumed due to the general expenses for the installation of the banks. The capacitor banks are made of modules having rated power of $150 \mathrm{kVAR}$ up to a maximum of 1200 $\mathrm{kVAR}$ ( 8 modules of $150 \mathrm{kVAR}$ ). The maximum number of

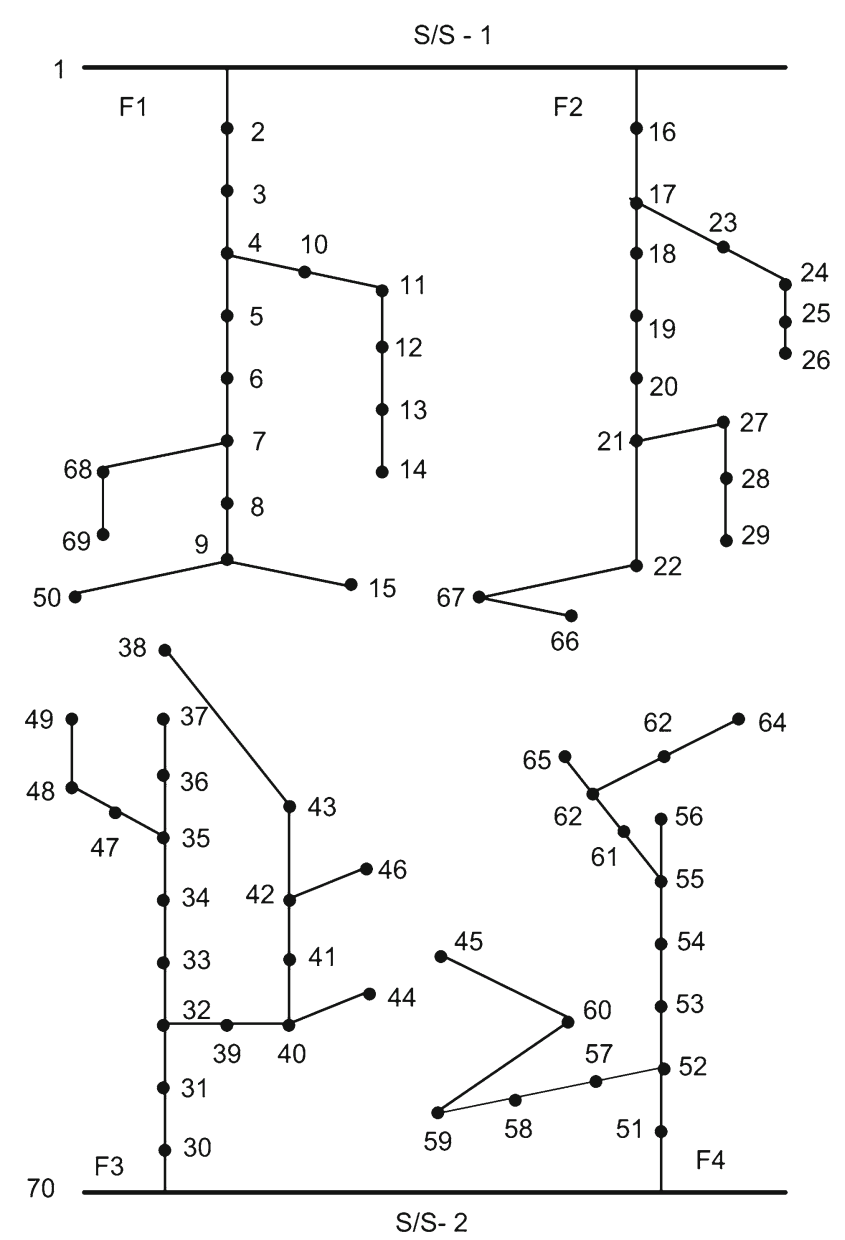

Fig. 14 Radial test system

compensated nodes, $n_{\text {comp.max }}$, is set first to 10 and then to 5 . The bus voltages can range between $\pm 6 \%$ compared to rated voltage. The parameters of the genetic algorithm have been identified heuristically; the values used in the applications are the following:

- population $=50$

- generations $=50$

- scaling factor $=2.00$

- probability of:

- simple mutation $=0.3$

- complete mutation $=0.3$ (probability mutation of a single bit $=0.5$ )

- simple mutation of a module $=0.3$ (probability mutation of a single bit $=0.5$ )

- complete mutation of the modules $=0.1$ (probability of mutation of a single bit $=0.1$ )

- module inversion $=0.1$

- position inversion $=0.1$

- node exchange $=0.1$

- module exchange $=0.1$ 
Table 1 Ratings of fixed and switchable capacitor banks at the nodes for $n_{\text {comp }}=10$

\begin{tabular}{lll}
\hline Bus no. & Fixed bank (kVAR) & Switched bank (kVAR) \\
\hline 12 & 150 & 150 \\
22 & 450 & 150 \\
43 & 300 & 150 \\
48 & 150 & 150 \\
50 & 300 & - \\
57 & 150 & 150 \\
65 & 150 & 300 \\
66 & 150 & 150 \\
\hline
\end{tabular}

- module crossover $=0.1$

- position crossover $=0.1$

- superposition from the initial bus $=0.1$

- superposition from the ending node $=0.1$

The optimal solution, for $n_{\text {comp, } \max }=10$, is shown in Table 1; the number of compensated nodes is 8 ; the energy losses in the uncompensated system are $2157 \mathrm{MWh}$, while in the compensated system they are only $1478 \mathrm{MWh}$. Under the hypotheses of minimum loss compensation ${ }^{4}$, the optimal solution is shown in Table 2; the number of compensated nodes is the maximum, the energy losses are equal to 1444 MWh, namely $2.3 \%$ less than the preceding, and total yearly cost is $\$ 114,123$.

With the aim of verifying the robustness of the algorithm, it has been run 50 times, evaluating the distribution of the probability of the overall cost of the compensated system in the attained solutions, (optimization executed considering all the costs); in Fig. 15, the frequency distribution is reported, having divided the range of values assumed by the cost into 7 intervals. The dispersion of the results is very limited, being contained between 98.64 and $101.52 \%$ of the average value; $68 \%$ of the values lie in the interval -0.13 to $+0.69 \%$ around the average value (taken equal to 100).

The optimal solution, for $n_{\text {comp, } \max }=5$, is shown in Table 3; number of compensated nodes is 5; energy losses are $1512 \mathrm{MWh}$ and total yearly cost is $\$ 109,304$; this latter is lower than that relevant to previous one $\left(n_{\text {comp }}=8\right)$ while energy losses are greater; this is due to the influence of the fixed cost for installation of bank; savings due to lower number of compensated buses overcome the higher cost due to energy losses. Nevertheless, in both cases energy losses and total yearly costs are very close.

\footnotetext{
4 The minimum losses compensation is equivalent to consider null the cost of capacitor banks; the value of losses in this condition may serve as a useful point of comparison to assess the quality of the solutions found when considering nonzero costs of compensation.
}

Table 2 Rated power of fixed and switchable capacitor banks at the nodes in the case of minimum losses compensation

\begin{tabular}{lll}
\hline Bus no. & Fixed bank (kVAR) & Switched bank (kVAR) \\
\hline 4 & 3000 & 150 \\
22 & 300 & 150 \\
30 & 150 & 150 \\
36 & 150 & 150 \\
41 & 450 & - \\
42 & - & 150 \\
44 & 150 & 150 \\
51 & 300 & - \\
62 & 150 & 150 \\
67 & 150 & 300 \\
\hline
\end{tabular}

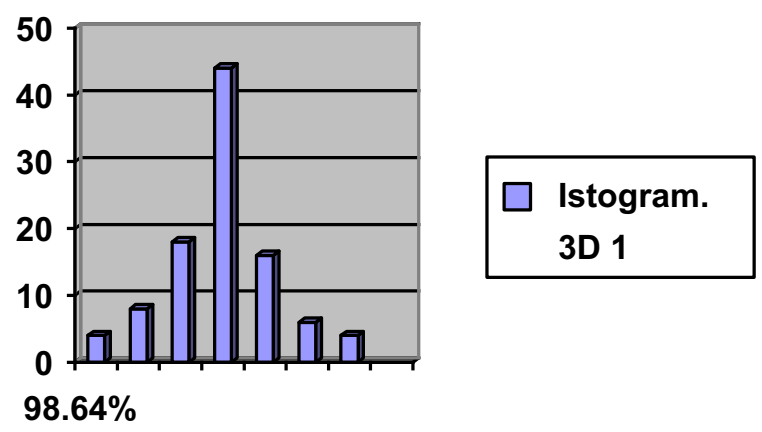

Fig. 15 Histogram of frequencies vs. cost values (for each solution the cost is expressed as a percentage of the mean value)

Table 3 Ratings of fixed and switchable capacitor banks at the nodes for $n_{\text {comp }}=5$

\begin{tabular}{lll}
\hline Bus no. & Fixed bank (kVAR) & Switched bank (kVAR) \\
\hline 30 & 450 & 0 \\
36 & 450 & 150 \\
63 & 150 & 450 \\
68 & 450 & 300 \\
69 & 150 & 300 \\
\hline
\end{tabular}

\section{Conclusions}

The problem of the optimal design of the compensation system for distribution networks, by means of fixed and modulated capacitor banks, with the aim of minimizing the annual cost of the loss of energy and annualized investment costs, has been used to put into evidence the features of a genetic algorithm designed for solving problems with three sets of unknowns (in the case of compensation: the locations, the rated power of the fixed part and of the switchable part of each bank). The representation of each individual of the population by means of a matrix, rather than by means of a string, allows developing a set of new genetic operators, thus widen- 
ing considerably the search space. The applications carried out have shown the good performance of the algorithm at identifying stable optimal solutions.

Acknowledgments This research was funded by University of Palermo as part of the FFR 2012 project.

\section{References}

1. Abul'Wafa, A.R.: Optimal capacitor allocation in radial distribution systems for loss reduction: a two stage method. Electr. Power Syst. Res. 95, 168-174 (2013)

2. Attia, A., El-Fergany, A., Abdelaziz, Y.: Capacitor placement for net saving maximization and system stability enhancement in distribution networks using artificial bee colony-based approach. Int. J. Electr. Power Energy Syst. 54, 235-243 (2014)

3. Augugliaro, A., Dusonchet, L., Favuzza, S., Ippolito, M.G., Riva Sanseverino, E.: Constrained robust multiobjective optimization for reactive design in distribution systems. In: Proceedings of PMAPS 2006-9th International Conference on Probabilistic Methods Applied to Power Systems, Stockholm (2006)

4. Carlisle, J.C., El-Keib Ivo, A.A.: A graph search algorithm for optimal placement of fixed and switched capacitors on radial distribution systems. IEEE Trans. Power Deliv. 15, 423-428 (2000)

5. Chaves da Silva Jr., I., Carneiro Jr., S., de Oliveira, E.J., de Souza, C.J., Rezende Pereira, J.L., Nepomuceno Garcia, P.A.: A heuristic constructive algorithm for capacitor placement on distribution systems. IEEE Trans. Power Syst. 23, 1619-1626 (2008)

6. Chang, C.F.: Reconfiguration and capacitor placement for loss reduction of distribution systems by ant colony search algorithm. IEEE Trans. Power Syst. 23, 1747-1755 (2008)

7. Das, D.: A fuzzy multiobjective approach for network reconfiguration of distribution systems. IEEE Trans. Power Deliv. 21, 202-209 (2006)

8. Eajal, A.A., El-Hawary, M.E.: Optimal capacitor placement and sizing in unbalanced distribution systems with harmonics consideration using particle swarm optimization. IEEE Trans. Power Deliv. 25, 1734-1741 (2010)

9. Gallego, R.A., Monticelli, A.J., Romero, R.: Optimal capacitor placement in radial distribution networks. IEEE Trans. Power Syst. 16, 630-637 (2001)

10. Guimarães, M.A.N., Castro, C.A., Romero, R.: Distribution systems operation optimisation through reconfiguration and capacitor allocation by a dedicated genetic algorithm. IEE Proc. Gener. Transm. Distrib. 4, 1213-1222 (2010)
11. Injeti, S.K., Thunuguntla, V.K., Shareef, M.: Optimal allocation of capacitor banks in radial distribution systems for minimization of real power loss and maximization of network savings using bioinspired optimization algorithms. Int. J. Electr. Power Energy Syst. 69, 441-455 (2015)

12. Haghifam, M.R., Malik, O.P.: Genetic algorithm-based approach for fixed and switchable capacitors placement in distribution systems with uncertainty and time varying loads. IEE Gener. Transm. Distrib. 1, 244-252 (2007)

13. Hsiao, Y.T., Chien, C.Y.: Optimisation of capacitor allocation using an interactive trade-off method. IEE Proc. Gener. Transm. Distrib. 148, 371-376 (2001)

14. Huang, S.J.: An immune-based optimization method to capacitor placement in a radial distribution system. IEEE Trans. Power Deliv. 15, 744-749 (2000)

15. Khodr, H.M., Olsina, F.G., De Oliveira-De, J.P.M., Yusta, J.M.: Maximum savings approach for location and sizing of capacitors in distribution systems. Electr. Power Syst. Res. 78, 1192-1203 (2008)

16. Ladjavardi, M., Masoum, M.A.S.: Genetically optimized fuzzy placement and sizing of capacitor banks in distorted distribution networks. IEEE Trans. Power Deliv. 23, 449-456 (2008)

17. Masoum, M.A.S., Ladjevardi, M., Jafarian, A., Fuchs, E.F.: Optimal placement, replacement and sizing of capacitor banks in distorted distribution networks by genetic algorithms. IEEE Trans. Power Deliv. 19, 1794-1801 (2004)

18. Mekhamer, S.F., Soliman, S.A., Moustafa, M.A., El-Hawary, M.E.: Application of fuzzy logic for reactive-power compensation of radial distribution feeders. IEEE Trans. Power Syst. 18, 206-213 (2003)

19. Mendes, A., Franca, P.M., Lyra, C., Pissarra, C., Cavellucci, C.: Capacitor placement in large-sized radial distribution networks. IEE Proc. Gener. Transm. Distrib. 152, 496-502 (2005)

20. Ng, H.N., Salama, M.M.A., Chikhani, A.Y.: Classification of capacitor allocation techniques. IEEE Trans. Power Deliv. 15, 387392 (2000)

21. Ng, H.N., Salama, M.M.A., Chikhani, A.Y.: Capacitor allocation by approximate reasoning: fuzzy capacitor placement. IEEE Trans. Power Deliv. 15, 393-398 (2000)

22. Sultana, S., Roy, P.K.: Optimal capacitor placement in radial distribution systems using teaching learning based optimization. Int. J. Electr. Power Energy Syst. 54, 387-398 (2014)

23. Venkatesh, B., Ranjan, R.: Fuzzy EP algorithm and dynamic data structure for optimal capacitor allocation in radial distribution systems. IEE Proc. Gener. Transm. Distrib. 153, 80-88 (2006) 